\title{
Analytical approximation solution for logistic delay differential equation
}

\author{
Nurul Atiqah Talib , Normah Maan*, Aminu Barde \\ Department of Mathematics, Faculty of Science, Universiti Teknologi Malaysia, 81310 UTM Johor Bahru, Johor, Malaysia \\ * Corresponding author: normahmaan@utm.my
}

\section{Article history}

Received 8 May 2019

Revised 14 August 2019

Accepted 20 November 2019

Published Online 15 June 2020

\begin{abstract}
Although the non-linear analytical techniques are fast developing, they still do not entirely satisfy mathematicians and engineers. Many researchers have conducted the study to find the analytical solution for the logistic delay differential equation. However, for the time lags occasion, it is quite hard and tough to achieve analytical solution due to its limitation, and thus, we can only expect the approximate analytical solution. This paper describes the approximate analytical techniques, homotopy analysis method (HAM), and homotopy perturbation method (HPM) in order to indicate their ability in solving the logistic delay differential equation. HAM is one of the better approaches that can be used for solving this equation. The use of HAM will lead to obtaining the series solution that contains an auxiliary parameter $h$ that can help to adjust and control the convergence and rate approximation for the series solution. Meanwhile, HPM is an analytical method with a combination of homotopy in topology and classical perturbation technique. Using the HPM technique, the logistic delay differential equation is reduced to a sufficiently simplified form, which usually becomes a linear equation that is easy to be solved. The comparison of numerical solution with $h$-values of HAM has shown the influence of parameter $h$ in the convergence of series solution. Using HAM and HPM, the relationship between the time-delay $\mathrm{T}$ and the population size is obtained. As a result, the higher the value of $\tau$, the steeper the gradient of the population size $x$. It is concluded that the parameter $h$ helps to adjust and control the convergence and rate approximation for the series solution of HAM. Laterally, the comparison between HAM and HPM with numerical method is done to show that both methods are relatively approximate to the exact solution. Moreover, homotopy perturbation method (HPM) is a special case of homotopy analysis method (HAM) when $H(t)=1$ and $h=-1$. Hence, using HAM and HPM techniques, two different kinds of series solutions of logistic delay differential equation are obtained.
\end{abstract}

Keywords: Logistic delay differential equation; homotopy analysis method (HAM); homotopy perturbation method (HPM).

\section{INTRODUCTION}

The logistic equation or called as the Verhulst model is a model of population growth produced by Pierre-Francois Verhulst in 1838. This population model is continuous with time. Diversity of biological growth has been improved for unpredicted, intraspecific population dynamics and many more. It is proven that most of the successful predictive growth models are derived from the extended forms of the classical logistic equation

$$
\frac{\mathrm{dN}(\mathrm{t})}{\mathrm{dt}}=\mathrm{rN}(\mathrm{t})\left[1-\frac{\mathrm{N}(\mathrm{t})}{\mathrm{K}}\right]
$$

where $N(t)$ is population density, $K$ is the carrying capacity and $r$ is population growth rate or known as Malthusian parameter.

A common approximation, for instance, like a growth for the initial period can be presented by the simple exponential growth model (Tsoularis and Wallace, 2002). However, for populations, this model considers that there is no intraspecific competition or predation. Therefore, the populations will persist to grow uninhibitedly and reduce to zero only with the existence of an initial growth reduction (Tsoularis and Wallace, 2002). However, there is a case where predation is fully disregarded, but the model still cannot adapt the reductions that caused by the intraspecific competition for environmental resources such as habitat and food (Tsoularis and Wallace, 2002). For example, when the plants reach a maturity phase, the physical features will approach a limiting dimension.

Moreover, this logistic equation plays a significant part in modeling world, involving more than one interacting population. In other word, the rate of growth for one or more of the interacting populations can satisfy the logistic equation with absence of the other populations (Julien, Lin and Gail, 2005). Many researchers that interested to explore on population dynamics are trying to establish the limitation and restriction for various populations that exist in this world. So far, some of the researches have been done for human populations in the USA by Pearl and Reed (1930) and Pearl et al. (1940), as well as in Canada by MacLean and Willard (1937).

Eventually, this interest slowly has became a popular subject for the mathematical analysis in many years. The topic includes the population dynamics, either discretely modeled or continuously modeled, especially for the large population (Tsoularis and Wallace, 2002). However, in reality, the rate of growth is not constant but declines after a period of time, and sometimes can cause a time delay, which is usually due to factors of environment and inherent genetic limitations (Hutchinson, 1948).

In 1948, Hutchinson (1948) suggested that the population growth in logistic equation is relevant and appropriate for the case when there is a lag in certain processes. Thus, this reformulation for the logistic 
equation that he suggested has gained a lot of attention from the mathematical community. The importance of this reality encourages Ruan (2006) to conduct the survey on these time-lag models, together with the discussion on distributed delays models in the form of integro differential equations. Besides known as the logistic delay equation, it is also called by the name of Hutchinson's equation, or Wright's equation if it undergoes a change of variable (Julien, Lin and Gail, 2005).

$$
\frac{d N(t)}{d t}=r N(t)\left[1-\frac{N(t-\tau)}{K}\right]
$$

where $\tau$ is the delay and a positive constant. The research on this logistic equations with the time delay has expanded very fast, including the research on the stability analysis, sensitivity analysis and also its application in population dynamics (see Rihan et al. (2014), Lakshmanan et al. (2014), Rihan et al. (2018) and Rihan et al. (2018)).

\section{DESCRIPTION OF THE METHOD}

\section{Basic ideas of HAM}

The homotopy analysis method (HAM) is first proposed by Liao in 1992 and has been widely used in handling and solving non-linear problems. The difficulties arise when most of perturbation techniques are depended on either small or large parameter, resulting them to be only suitable to apply on weakly non-linear problems. Homotopy Analysis Method (HAM) has been successfully proven to overcome the obstacles (Liao, 1997). Despite of being different from all perturbation and non-perturbation methods, HAM conveniently provides us a better way to adjust and control the convergence region and rate of approximation series. The convergence-control parameter, $h$ is a nonphysical variable which provides a pleasant way to verify and naturally enforce the solution series to be converged. There is a rapid growth of this research as can be seen in the literatures such as in Yin et al. (2015), Olvera et al. (2015), and Abolhasani et.al (2016). Abolhasani et.al (2016) introduced a new method called modified Homotopy perturbation method for solving delay differential equations based on the new Homotopy perturbation method and Pade approximation, which is very useful to control the convergence region of approximate solutions. This method solves neutral functional differential equations with proportional delays and the multi-pantograph delay equations, in which the results are compared with that of the Homotopy analysis method, new Homotopy analysis method, and variational iteration method. However, this method is not suitable to be applied in solving logistic delay differential equation.

The basic idea of HAM is explained in more detail manner in the next section.

\section{Zeroth-order deformation equation} below

First, consider one non-linear equation in general form as shown

$$
\mathcal{N}[x(t)]=0 .
$$

Let $x_{0}(t)$ be an initial guess of the exact solution $x(t)$. Since $h \neq 0$ is an auxiliary parameter and $H(t) \neq 0$ is an auxiliary function, thus $\mathcal{L}$ is an auxiliary linear operator with the property

$\mathcal{L}[\Phi(t ; q)]=0 \quad$ when $\Phi(t ; q)=0$.

Using $q \epsilon[0,1]$ as an embedding parameter, we build a homotopy equation,

$$
\begin{aligned}
\mathcal{H}\left[\Phi(t ; q) ; x_{0}(t),\right. & H(t), h, q] \\
& =(1-q)\left\{\mathcal{L}\left[\Phi(t ; q)-x_{0}(t)\right]\right\} \\
& -q h H(t) \mathcal{N}[\Phi(t ; q)]=0 .
\end{aligned}
$$

The zero order deformation equation is as below

$$
(1-q)\left\{\mathcal{L}\left[\Phi(t ; q)-x_{0}(t)\right]\right\}=q h H(t) \mathcal{N}[\Phi(t ; q)]
$$

and stated that $\Phi(t ; q)$ is the solution that relies on the auxiliary linear operator $\mathcal{L}$, initial guess $x_{0}(t)$, auxiliary parameter $h$, auxiliary function $H(t)$ and embedding parameter $q$.

If the value $q=0$ and $q=1$ with $h \neq 0, H(t) \neq 0$, the equation (2) becomes

$$
\begin{aligned}
& \Phi(t ; 0)=x_{0}(t) \\
& \Phi(t ; 1)=x(t) .
\end{aligned}
$$

The $m$ th order deformation derivatives-is defined as,

$$
x_{0}^{[m]}(t)=\left.\frac{\partial^{m} \Phi(t ; q)}{\partial q^{m}}\right|_{q=0} .
$$

Using Taylor's theorem, $\Phi(t ; q)$ can be expanded in power series of $q$ to become

$$
\Phi(t ; q)=\Phi(t ; 0)+\sum_{m=1}^{+\infty} \frac{x_{0}^{[m]}(t)}{m !} q^{m} .
$$

Thus, $\Phi(t ; q)$ becomes

$$
\Phi(t ; q)=x_{0}(t)+\sum_{m=1}^{+\infty} x_{m}(t) q^{m}
$$

where

$$
x_{m}(t)=\frac{x_{0}^{[m]}(t)}{m !}=\left.\frac{1}{m !} \frac{\partial^{m} \Phi(t ; q)}{\partial q^{m}}\right|_{q=0} .
$$

As $q=1$, this leads to assumption for the solution series,

$$
\Phi(t ; 1)=x(t)=x_{0}(t)+\sum_{m=1}^{+\infty} x_{m}(t) .
$$

The above expression shows the relationship between the exact solutions $x(t)$ and the initial approximation $x_{0}(t)$ by the terms of $x_{m}(t)$. Next, the term $x_{m}(t)$ will be discovered by the higher-order deformation equation as described below.

\section{Higher-order deformation equation}

Defining the vector,

$\vec{x}_{n}(t)=\left\{x_{0}(t), x_{1}(t), \ldots, x_{n-1}(t), x_{n}(t)\right\}$.

The zero order deformation equation is differentiated by $m$ times with respect to $q$, the embedding parameter. By dividing it by $m$ ! and set $q=0$, the $m_{\text {th }}$ order deformation equation is produced

$$
\mathcal{L}\left[x_{m}(t)-\chi_{m} x_{m-1}(t)\right]=h H(t) R_{m}\left[\vec{x}_{m-1}(t)\right]
$$

where

$$
R_{m}\left[\vec{x}_{m-1}(t)\right]=\left.\frac{1}{(m-1) !} \frac{\partial^{m-1} \mathrm{~N}[\Phi(t ; q)]}{\partial q^{m-1}}\right|_{q=0} .
$$

and $\chi_{m}$ is introduced by

$$
\chi_{m}= \begin{cases}1 & \text { when } m>1 \\ 0 & \text { otherwise }\end{cases}
$$

Rearranging equation (4), we obtain

$$
x_{m}(t)=\chi_{m} x_{m-1}(t)+h \mathcal{L}^{-1}\left\{H(t) R_{m}\left[\vec{x}_{m-1}(t)\right]\right\} .
$$

Finally, the solution series of HAM is obtained as follows,

$$
x(t)=\sum_{m=0}^{+\infty} x_{m}(t) .
$$

The convergence of the series solution is essential to be proved as provided by Liao (2003). A series solution is considered as a good one if it converges rather than diverges. One of the main factors that influence the convergence of the series solution is the type of base function used to express the solution. Thus, the base functions are expected to be more likely to resemble the behavior of the actual 
solution, which will provide a better outcome. These auxiliary linear operator $\mathcal{L}$, auxiliary function $H(t)$, auxiliary parameter $h$ and the initial approximation $x_{0}(t)$ choices are also the important keys to determine the convergence of the series solution. In order to determine the optimum value of $h$, the graph of so-called $h$-curves of the solution is plotted. These curves are plotted by sums of $x_{m}(t)$ and/or their first derivatives that evaluated at a specific chosen $t$ value against the auxiliary parameter $h$.

\section{Basic ideas of HPM}

The homotopy perturbation method (HPM) is first proposed by J. Huan $\mathrm{He}$, the Chinese mathematician. Basically, the perturbation techniques is based on the existence of small or large parameters-called as perturbation quantity. This small parameter assumption restricts the application of perturbation techniques since most of the non-linear problems do not consist of small parameter (He, 2000). Moreover, the determination of small parameter seems to be important, otherwise it will lead to bad results (He, 2000). Hence, this is where the homotopy perturbation method (HPM) is implemented to give the approximate analytical solutions for the non-linear problems. The main idea is by introducing a homotopy parameter, $p$ which corresponded to values from 0 to 1 . When the value $p$ is 0 , the equation reduces to a simplified form, which normally becomes a linear equation. As $p$ is slowly increased to $p=1$, the equation goes under a chronology of deformations. Up until $p=1$, the equation follows the original equation and becomes the last stage of deformation to give the analytical solution. Next, the solution is expanded into a series of $p$ and then solved in sequence according to the power of $p$. HPM is known for its remarkable features, which it sufficiently requires several perturbation terms to gain a reasonably precise solution.

To illustrate the basic idea of the HPM, consider one non-linear differential equation,

$$
A(u)-f(\boldsymbol{r})=0, \quad \boldsymbol{r} \in \Omega,
$$

with the following boundary condition

$$
B\left(u, \frac{\partial u}{\partial n}\right)=0, \quad \boldsymbol{r} \in \Gamma,
$$

where $A$ is a general differential operator, $B$ is a boundary operator, $\boldsymbol{r}$ is a spatial independent variables, $f(\boldsymbol{r})$ is a known analytical function and $\Gamma$ is the boundary of the domain $\Omega$. Generally, operator $A$ can be splitted into two parts that are is $L$ and $N$, where $L$ is linear and $N$ is non-linear.

$$
L(u)+N(u)-f(\boldsymbol{r})=0 .
$$

We construct a homotopy equation with $p \in[0,1]$ as an embedding parameter and $u_{0}$ as an initial approximation,

$$
\begin{aligned}
& H(v, p)=(1-p)\left[L(v)-L\left(u_{0}\right)\right]+p[A(v)-f(\boldsymbol{r})]=0, \\
& p \in[0,1], \boldsymbol{r} \in \Omega .
\end{aligned}
$$

If

$p$ is set to 0 and 1 , the equation (5) is obtained as follows :

$$
\begin{gathered}
H(v, 0)=L(v)-L\left(u_{0}\right)=0, \\
H(v, 1)=A(v)-f(\boldsymbol{r})=0 .
\end{gathered}
$$

The above process of changing $p$ from zero to unity is just the displacement of $v(\boldsymbol{r}, p)$ from $u_{0}(\boldsymbol{r})$ to $u(\boldsymbol{r})$. In topology, this is called deformation, while $L(v)-L\left(u_{0}\right)$ and $A(v)-f(\boldsymbol{r})$ are called homotopies.

The embedding parameter, $p$ is used as a small parameter and assumes that the solution can be written as a power series in $p^{\circ}$

$$
v=v_{0}+p v_{1}+p^{2} v_{2}+\cdots
$$

By setting $p=1$, the approximation solution of HPM is obtained as follows,

$$
u=\lim _{p \rightarrow 1} v=v_{0}+v_{1}+v_{2}+\cdots
$$

HOMOTOPY ANALYSIS METHOD (HAM) AND HOMOTOPY PERTURBATION METHOD (HPM) FOR SOLVING LOGISTIC DELAY DIFFERENTIAL EQUATION

We use HAM and HPM to solve the logistic delay differential equation as shown below,

$$
\begin{gathered}
\frac{d x(t)}{d t}-r x(t)+\mu x(t) x(t-\tau)=0, \text { where } \mu=\frac{r}{k^{\prime}} \\
x(0)=\alpha
\end{gathered}
$$

where $x$ is the population size, $\alpha$ is the initial amount, $k$ is the carrying capacity and $r$ is the population growth rate.

\section{Homotopy analysis method (HAM) for solving logistic delay differential equation}

For this delay problem, we choose $\left\{e_{n}(t) \mid n=0,1,2 \ldots\right\}$ as a base function. Thus, the solution can be written as

$$
x_{m}(t)=\sum_{m=0}^{M} c_{m} e_{m}(t)
$$

where $c_{m}$ is constant. So, for the initial guess, we choose

$$
x_{0}(t)=\alpha e^{r t} .
$$

The auxiliary linear operator

with property

$$
\mathcal{L}[x(t ; q)]=\frac{\partial x(t ; q)}{\partial t}
$$

$$
\mathcal{L}[c]=0
$$

where $c$ is the integral constant.

Defining the nonlinear operator,

$$
\mathcal{N}[x(t ; q)]=\frac{\partial x(t ; q)}{\partial t}-r x(t ; q)+\mu x(t ; q) x(t-\tau ; q) .
$$

Note that the solution series we are looking for

$$
x(t ; 1)=x(t)=x_{0}(t)+\sum_{m=1}^{+\infty} x_{m}(t) .
$$

For the term $x_{m}(t)$, we use the $m$ th order deformation equation,

where

$$
\mathcal{L}\left[x_{m}(t)-\chi_{m} x_{m-1}(t)\right]=h R_{m}\left[\vec{x}_{m-1}(t)\right]
$$

$$
R_{m}\left[\vec{x}_{m-1}(t)\right]=x_{m-1}^{\prime}(t)-r x_{m-1}(t)+\mu \sum_{i=0}^{m-1} x_{i}(t) x_{m-1-i}(t-\tau)
$$

and

$$
\chi_{m}=\left\{\begin{array}{lc}
1 & \text { when } m>1 \\
0 & \text { otherwise. }
\end{array}\right.
$$

By algebraic manipulation, $m$ th order deformation equation of (7) for $m \geq 1$, becomes

$$
x_{m}(t)=\chi_{m} x_{m-1}(t)+h \mathcal{L}^{-1}\left\{R_{m}\left[\vec{x}_{m-1}(t)\right]\right\} .
$$

We solve equation (7) with initial guess (6). Thus, we successively obtain

$x_{0}(t)=\alpha e^{r t}$

$x_{1}(t)=\frac{1}{2 r} h \mu \alpha^{2} e^{-r \tau} e^{2 r t}$ 


$$
\begin{gathered}
x_{2}(t)=\frac{1}{4 r} h \mu \alpha^{2} e^{-r \tau}(h+2) e^{2 r t}+\frac{1}{6 r^{2}} h^{2} \mu^{2} \alpha^{3}\left(e^{-2 r \tau}\right. \\
\left.+e^{-3 r \tau}\right) e^{3 r t} \\
x_{3}(t)=\frac{1}{8 r} h \mu \alpha^{2} e^{-r \tau}(h+2)^{2} e^{2 r t}+\frac{1}{36 r^{2}} h^{2} \mu^{2} \alpha^{3}\left(e^{-2 r \tau}\right. \\
\left.+e^{-3 r \tau}\right)(7 h+12) e^{3 r t} \\
+\frac{1}{48 r^{3}} h^{3} \mu^{3} \alpha^{4}\left(2 e^{-3 r \tau}+5 e^{-4 r \tau}+2 e^{-5 r \tau}\right. \\
\left.+2 e^{-6 r \tau}\right) e^{4 r t}
\end{gathered}
$$

$x_{4}(t)=\frac{1}{16 r} h \mu \alpha^{2} e^{-r \tau}(h+2)^{2}(1+2 h) e^{2 r t}$

$$
\begin{aligned}
& +\left(\frac { 1 } { 2 1 6 r ^ { 2 } } h ^ { 2 } \mu ^ { 2 } \alpha ^ { 3 } \left(e^{-2 r \tau}\right.\right. \\
& \left.+e^{-3 r \tau}\right)(7 h+12)(6 h+4) \\
& \left.+\frac{1}{24 r^{2}} h \mu^{2} \alpha^{3}(h+2)^{2}\left(e^{-2 r \tau}+e^{-3 r \tau}\right)\right) e^{3 r t} \\
& +\left(\frac{1}{64 r^{3}} h^{3} \mu^{3} \alpha^{4}(1\right. \\
& \left.+\frac{4}{3} h\right)\left(2 e^{-3 r \tau}+5 e^{-4 r \tau}+2 e^{-5 r \tau}+2 e^{-6 r \tau}\right) \\
& +\frac{7}{144 r^{3}} h^{3} \mu^{3} \alpha^{4}\left(e^{-3 r \tau}+\frac{16}{7} e^{-4 r \tau}+e^{-5 r \tau}\right. \\
& \left.+e^{-6 r \tau}\right) \\
& +\frac{1}{12 r^{3}} h^{2} \mu^{3} \alpha^{4}\left(e^{-3 r \tau}+\frac{5}{2} e^{-4 r \tau}+e^{-5 r \tau}\right. \\
& \left.\left.+e^{-6 r \tau}\right)\right) e^{4 r t} \\
& +\frac{1}{120 r^{4}} h^{3} \mu^{4} \alpha^{5}\left(e^{-4 r \tau}+\frac{9}{2} e^{-5 r \tau}+5 e^{-6 r \tau}\right. \\
& \left.+4 e^{-7 r \tau}+\frac{5}{2} e^{-8 r \tau}+e^{-9 r \tau}+e^{-10 r \tau}\right) e^{5 r t} .
\end{aligned}
$$

Finally, the solution of series form is given by

$$
x(t)=x_{0}(t)+x_{1}(t)+x_{2}(t)+x_{3}(t)+x_{4}(t)+\cdots
$$

\section{Homotopy perturbation method (HPM) for solving logistic delay differential equation}

Construct the homotopy equation as follows,

$$
\begin{aligned}
H(X, p)=(1-p)[ & \left.X^{\prime}(t)-r X(t)-x_{0}^{\prime}(t)+r x_{0}(t)\right] \\
& +p\left[X^{\prime}(t)-r X(t)+\mu X(t) X(t-\tau)\right]=0 .
\end{aligned}
$$

Assume the solution has the form,

$$
\begin{gathered}
X(t)=X_{0}(t)+p X_{1}(t)+p^{2} X_{2}(t)+p^{3} X_{3}(t)+p^{4} X_{4}(t) \\
+O\left(p^{5}\right) \\
\begin{array}{c}
X^{\prime}(t)=X^{\prime}{ }_{0}(t)+p \\
X^{\prime}{ }_{1}(t)+p^{2} X^{\prime}{ }_{2}(t)+p^{3} X^{\prime}{ }_{3}(t)+p^{4} X^{\prime}{ }_{4}(t) \\
+O\left(p^{5}\right) .
\end{array}
\end{gathered}
$$

Substituting equations (11) and (12) into equation (10), the equation becomes,

$\left[X^{\prime}{ }_{0}(t)+p X^{\prime}{ }_{1}(t)+p^{2} X^{\prime}{ }_{2}(t)+p^{3} X^{\prime}{ }_{3}(t)+p^{4} X^{\prime}{ }_{4}(t)\right]-r\left[X_{0}(t)+\right.$ $\left.p X_{1}(t)+p^{2} X_{2}(t)+p^{3} X_{3}(t)+p^{4} X_{4}(t)\right]-x_{0}^{\prime}(t)+r x_{0}(t)+$ $p x_{0}^{\prime}(t)-\operatorname{prx}_{0}(t)+p \mu\left[X_{0}(t)+p X_{1}(t)+p^{2} X_{2}(t)+p^{3} X_{3}(t)+\right.$ $\left.p^{4} X_{4}(t)\right]\left[X_{0}(t-\tau)+p X_{1}(t-\tau)+p^{2} X_{2}(t-\tau)+p^{3} X_{3}(t-\tau)+\right.$ $\left.p^{4} X_{4}(t-\tau)\right]=0$.

By using some algebraic manipulations to equation (13) and collecting the terms according to the identical powers of $p$, the following equations are obtained,

$$
\begin{aligned}
& p^{0}: X^{\prime}{ }_{0}(t)-r X_{0}(t)-x_{0}(t)+r x_{0}(t)=0 \\
& p^{1}: X^{\prime}{ }_{1}(t)-r X_{1}(t)+x_{0}(t)-r x_{0}(t)+\mu X_{0}(t) X_{0}(t-\tau)=0
\end{aligned}
$$

$$
\begin{aligned}
& p^{2}: X^{\prime}{ }_{2}(t)-r X_{2}(t)+\mu X_{0}(t) X_{1}(t-\tau)+\mu X_{1}(t) X_{0}(t-\tau)=0 \\
& p^{3}: X^{\prime}{ }_{3}(t)-r X_{3}(t)+\mu X_{0}(t) X_{2}(t-\tau)+\mu X_{1}(t) X_{1}(t-\tau) \\
& +\mu X_{2}(t) X_{0}(t-\tau)=0 \\
& p^{4}: X^{\prime}{ }_{4}(t)-r X_{4}(t)+\mu X_{0}(t) X_{3}(t-\tau)+\mu X_{1}(t) X_{2}(t-\tau) \\
& +\mu X_{2}(t) X_{1}(t-\tau)+\mu X_{3}(t) X_{0}(t-\tau)=0 .
\end{aligned}
$$

Solve the equations of $p^{0}, p^{1}, p^{2}, p^{3}$ and $p^{4}$ in sequence, together with the given initial condition to obtain $X_{0}(t), X_{1}(t), X_{2}(t), X_{3}(t)$ and $X_{4}(t)$.

$$
\begin{aligned}
& X_{0}(t)=\alpha \\
& X_{1}(t)=\frac{\mu \alpha^{2}}{r}-\alpha+ e^{r t}\left(-\frac{\mu \alpha^{2}}{r}+\alpha\right) \\
& X_{2}(t)=\frac{1}{r}\left[\mu \alpha^{2}(\mu \alpha-r)\left(\frac{2 e^{-r t}}{r}+t+t e^{-r \tau}\right)-\frac{2 \mu \alpha^{2}(\mu \alpha-r)}{r}\right] e^{r t} \\
& X_{3}(t)=\frac{\mu \alpha^{2}(-\mu \alpha+r) e^{r t}}{r^{2}}\left[\frac{(\mu \alpha-r) e^{r(t-\tau)}}{r}\right. \\
&+e^{-r \tau}\left[\mu \alpha\left(-3 t-r \tau t+r t^{2}\right)+r t\right] \\
&+ r u \alpha e^{-2 r \tau}\left(\frac{t^{2}}{2}-\tau t\right)-\frac{(5 u \alpha-r) e^{-r t}}{r} \\
&\left.+u \alpha\left(\frac{r t^{2}}{2}-3 t\right)+r t\right]+C e^{r t} \\
& \\
& X_{4}(t)=-\frac{\alpha^{3} u^{2}(}{2}u \alpha+r)\left[\left[\left(\left(u \alpha t^{2}+4 t\right) r^{2}+(-14-10 u \alpha t) r\right.\right.\right. \\
& 2 e^{3 r} r^{4} \\
&+28 u \alpha) e^{r t}+12 r^{2} t+(-28 u \alpha t+14) r \\
&-28 u \alpha] e^{3 r \tau} \\
&+\left[((-u \alpha+r)(4+r \tau-2 r t)) e^{2 r t}\right. \\
&+\left(((3 t-\tau)(t-\tau) u \alpha-4 \tau+8 t) r^{2}\right. \\
&+(-16-(22 t-12 \tau) u \alpha) r+38 u \alpha) e^{r t} \\
&+\left(3 \tau-u \alpha \tau^{2}\right) r^{2}+(12-11 u \alpha \tau) r \\
&-34 u \alpha] e^{2 r \tau} \\
&+\left[(-u \alpha+r)(1+r \tau-r t) e^{2 r t}\right. \\
&+\left((u \alpha(t-2 \tau)(3 t-2 \tau)-4 \tau+4 t) r^{2}\right. \\
&+(-2+16 \tau-14 t) u \alpha r) e^{r t}+\left(3 \tau-4 u \alpha \tau^{2}\right) r^{2} \\
&+(1-15 u \alpha \tau) r-11 u \alpha] e^{r \tau}+(\mu \alpha-r) e^{2 r t} \\
&+\mu \alpha\left(2+(t-\tau)(t-3 \tau) r^{2}+(4 \tau r-2 r t)\right) e^{r t} \\
&\left.-3 r^{2} u \alpha \tau^{2}+(1-4 u \alpha \tau) r-3 u \alpha\right] .
\end{aligned}
$$

Finally, the solution becomes

$$
\begin{aligned}
x(t)=\lim _{p \rightarrow 1} X(t)= & \lim _{p \rightarrow 1}\left(X_{0}(t)+p X_{1}(t)+p^{2} X_{2}(t)+p^{3} X_{3}(t)\right. \\
& \left.+p^{4} X_{4}(t)+\cdots\right)
\end{aligned}
$$

\section{NUMERICAL RESULT}

In this work, the algebraic computations and drawings of graphics are carried out by using MATLAB software. The valid region of $h$ curves is a horizontal line segment, as pointed out by Liao (1992). Based on calculation, the horizontal line can be calculated to obtain the values or range of parameter $h$ using the gradient of both $x(0)$ and $x^{\prime}(0)$ which is approximately approached the zero. Thus, the valid region of $h$ based on Fig. 1 is taken in the range of $-2.1<h<-0.2$. To validate the accuracy of the HAM solution with different values of $h$, we define the residual error for the series as

$$
E=S^{\prime}(t)-r S(t)+\mu S(t) S(t-\tau)
$$

where $S(t)$ is the HAM series solution (Alomari, Noorani and Nazar, 2009). Hence, from Fig. 2, we can conclude that $h=-1.5$ that consisted with the minimum error compared to other values of $h$ 


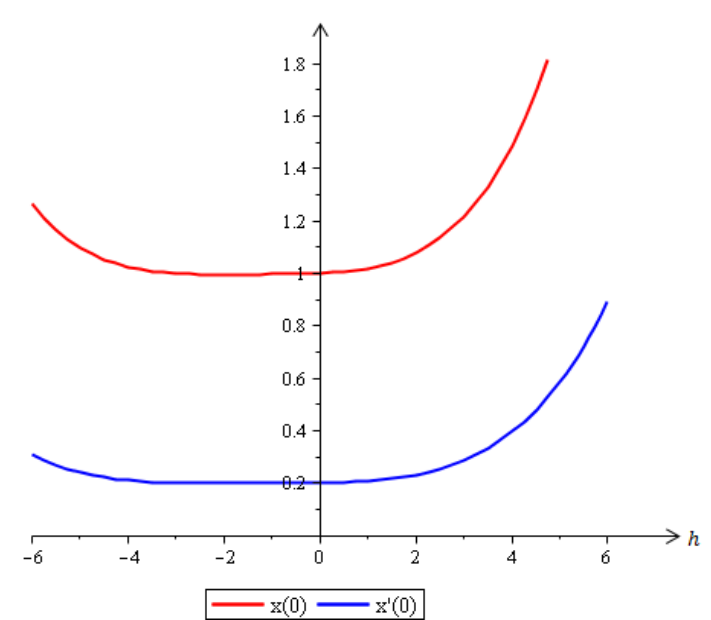

Fig. 1 The $h$-curves of 4 th-order approximation with values $\alpha=1, r=$ $0.2, k=200$, and $\tau=0.5$

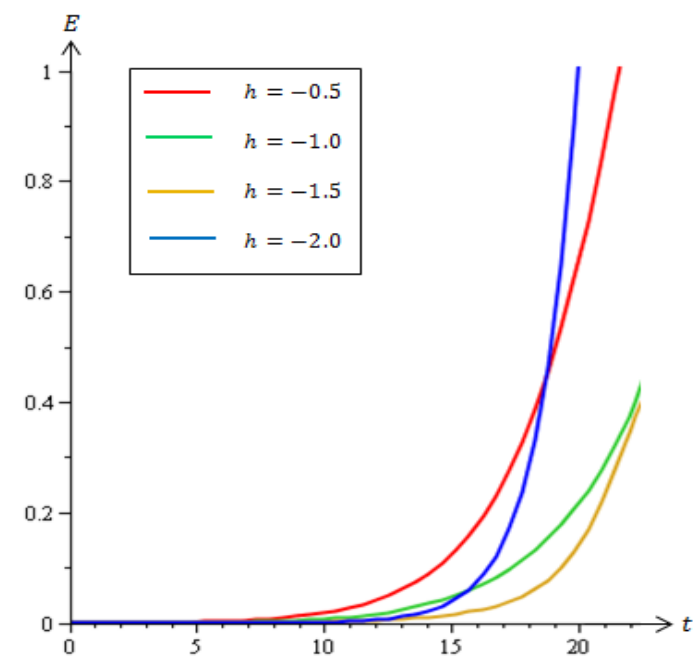

Fig. 2 The residual error of 4 th order approximation solution of $x(t)$ for different values of $h$ with values $\alpha=1, r=0.2, k=200$, and $\tau=0.5$

From the results obtained in Fig. 3 and Fig. 4, we can see that the time-delay $\tau$ imposes a great influence on the global dynamics of the system where it varies as $\tau$ varies. When $\tau=0$, both figures (Fig. 3 and 4) have different patterns. This may due to the convergence of the series solution which resulted in different regions.

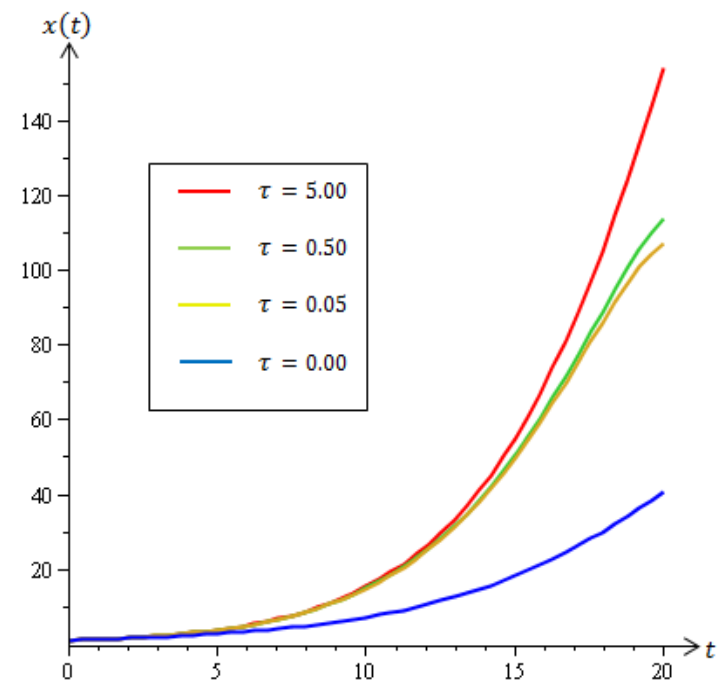

Fig. 3 The 4th order approximation solution of HAM for different values of $\tau$ with values $\alpha=1, r=0.2, k=200$, and $h=-1.5$.

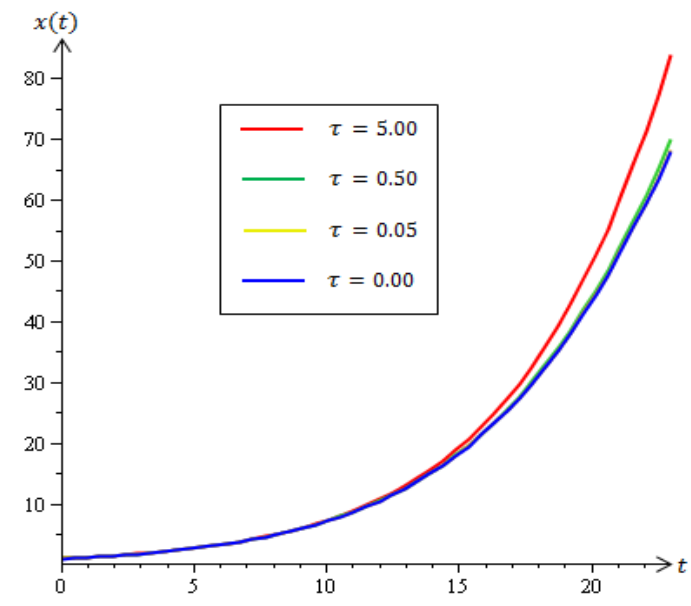

Fig. 4 The 4th order approximation solution of HPM for different values of $\tau$ with values $\alpha=1, r=0.2$, and $k=200$.

Both homotopy analysis method (HAM) and homotopy perturbation method (HPM) are based on the homotopy, which is a basic concept of topology. Hence, we need to compare the solution with the numerical method as shown by Fig. 5. For this logistic delay differential equation, we use explicit Runge-Kutta method to obtain the numerical solution. From Fig. 6, we can see obviously that the best $h$ value in this case will be $h=-1.5$.

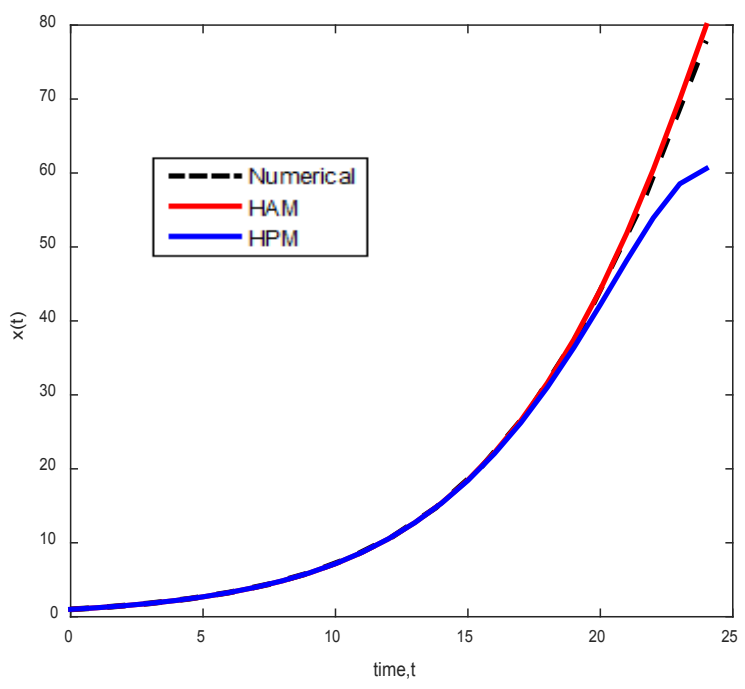

Fig. 5 The comparison of numerical result with HAM and HPM of 4th order approximation solution.

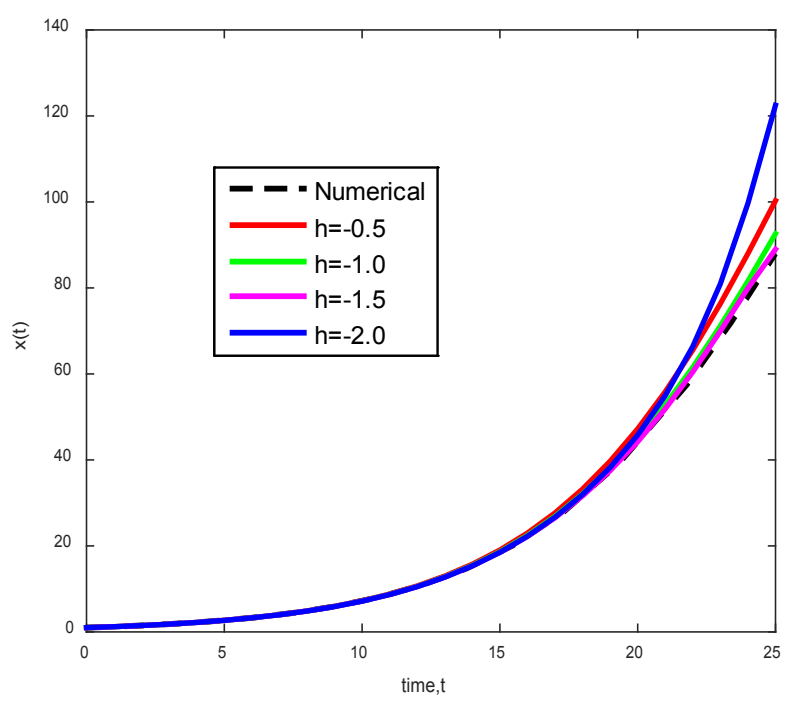

Fig. 6 The comparison of numerical result and 4th order approximation of HAM solution for different values of $h$ with values $\alpha=1, r=0.2, \tau=$ 0.5 and $k=200$. 
Furthermore, HPM is considered as a special case of HAM when the auxiliary parameter, $h=-1$ (Liao, 2005). Research carried out by Chowdhury, Hashim \& Abdulaziz (2009) for the purely non-linear fintype problems has shown that HPM is a special case of HAM when $h=$ -1 . In our case, for this logistic delay differential equation, the figure of 4th order approximation solution of HPM and 4th order approximation solution of HAM when $h=-1$ is plotted in Fig. 7 to show the agreement of the finding and thus, HPM is a special case of HAM when $h=-1$. The absolute error of the solutions from HAM and HPM of 4th order approximation solution of $x(t)$ at time $t$ is shown in Table 1.

Table 1 Absolute error of HAM solution and HPM solution of 4th order approximation solution of $x(t)$ at time $t$.

\begin{tabular}{ccc}
\hline time, $\boldsymbol{t}$ & $\begin{array}{c}\text { Absolute error } \\
\text { between HAM and } \\
\text { numerical }\end{array}$ & $\begin{array}{c}\text { Absolute error } \\
\text { between HPM and } \\
\text { numerical }\end{array}$ \\
\hline 0 & 0.0042 & 0 \\
2 & 0.0061 & 0 \\
4 & 0.0085 & 0.0001 \\
6 & 0.0115 & 0.0002 \\
8 & 0.0147 & 0.0003 \\
10 & 0.0145 & 0.0023 \\
12 & 0.0075 & 0.0032 \\
14 & 0.0189 & 0.0046 \\
16 & 0.0770 & 0.0773 \\
18 & 0.1994 & 0.3980 \\
20 & 0.4799 & 1.5642 \\
\hline
\end{tabular}

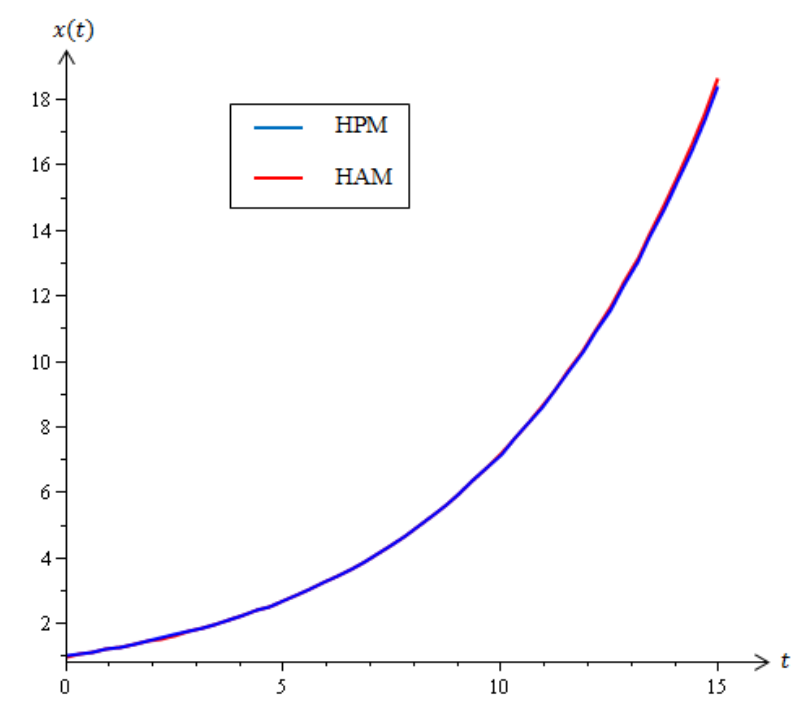

Fig. 7 The comparison of 4 th order approximation solution of HPM and 4th order approximation solution of $\operatorname{HAM}$ with value $h=-1$.

\section{CONCLUSION}

The aim of this research is to apply the homotopy analysis method (HAM) and homotopy perturbation method (HPM) in the logistic delay differential equation. After several filtrations throughout the residual errors calculated, we conclude that $h=-1.5$ is the best option for the HAM. The accuracy of HAM and HPM for this problem is considered through up until $t=16$ with the absolute error below than $1.0 \times 10^{-1}$. However, if the time $t$ increases, the series solution starts to diverge. Hence, the application of HAM and HPM on logistic delay differential equation can produce the converged series solution for $t \leq 16$. Using the HAM and HPM methods, we have succeeded in developing two different approximations to the problem considered in this study and laterally made the comparison with the numerical result.

This time-delay related problem should not be ignored even though it has its own difficulties in finding the analytical solutions. Although there is a better numerical algorithm, the analytical solutions still have their own advantages. Despite the fact that numerical techniques have been leading for several decades, there are still a lot of attempts to create and develop the new method in order to gain the analytical solutions which are fairly approximate to the exact solution.

\section{ACKNOWLEDGEMENT}

The authors were thankful to Research Management Center (UTM) and Ministry of Higher Education (MOHE), Malaysia for financial support through research grants of vote 4L854 and 4F797.

\section{REFERENCE}

Abolhasani, M., Ghaneai, H. and Heydari, M. 2016. Modified homotopy perturbation method for solving delay differential equations. Appl. Sci. Rep $16(2), 89-92$

Alomari A. K., Noorani M. S. M. and Nazar, R. 2009. Solution of delay differential equation by means of homotopy analysis method. Acta Appl Math., 108(2), 395-412.

Chowdhury M. S. H, Hashim I., and Abdulaziz O. 2009. Comparison of homotopy analysis method and homotopy perturbation method for purely nonlinear fin-type problems. Communications in Nonlinear Science and Numerical Simulation, 14(2), 371-378.

He, J. H. 2000. A coupling method of a homotopy perturbation technique for nonlinear problems. International Journal of Nonlinear Mechanics, 35(1), 37-43.

Hutchinson, G. E. 1948. Circular causal systems in ecology. Annals New York Academy of Sciences, 50(4), 221-246.

Julien, A., Lin, W., and Gail, S. K. W. 2005. An alternative formulation for a delayed logistic equation. Journal of Theoretical Biology, 241(1), 109-119.

Lakshmanan, S., Rihan, F. A., Rakkiyappan, R., and Park, J. H. 2014. Stability Analysis of the differential genetic regulatory networks model with timevarying delays and markovian jumping parameters. Nonlinear Analysis: Hybrid Systems, 14, 1-15.

Liao, S. J. 1992. The proposed homotopy analysis technique for the solution of nonlinear problems. International Journal of Non-Linear Mechanics, 34(4), 759-778.

Liao, S. J. 1997. Boundary element method for general nonlinear differential operators. Engineering Analysis with Boundary Element, 20(2), 91-99.

Liao, S. J. 2003. Beyond Perturbation: Introduction to Homotopy Analysis Method. Chapman and Hall/CRC. Boca Raton.

Liao, S. J. 2005. Comparison between the homotopy analysis method and homotopy perturbation method. Applied Mathematics and Computation, 169(2), 1186-1194.

MacLean, M. C. and Willard Turner, A. 1937. The logistic curve applied to canada's population. Canadian Journal Economy Political Sciences, 3(2), 241-248.

Olvera, D., El' 1as-Zún iga, A., Lo' pez de Lacalle, L. and Rodr' 1 guez, C. 2015. Approximate solutions of delay differential equations with constant and variable coefficients by the enhanced multistage homotopy perturbation method. Abstract and Applied Analysis. Hindawi, vol. 2015.

Pearl, R. and Reed, L. J. 1930. The logistic curve and the census count of 1930. Science, 72 (1868), 399-401.

Pearl, R., Reed, L. J. and Fish, J. F. 1940. The logistic curve and the census count of 1940. Science 92 (2395), 486-488.

Rihan, F. A., Abdel rahman, D.H., Lakshmanan, S. and Alkhajeh, A. S. 2014. A time delay model of tumor-immune system interactions: Global dynamics, parameter estimation, sensitivity analysis. Applied Mathematics and Computation, 232, 606-623.

Rihan, F. A., Azamov, A. A., Al Sakaji, H. J. 2018, An inverse problem for delay differential equations: Parameter estimation, nonlinearity and sensitivity. Applied Matematics and Information Science, 12(1), 63-74.

Rihan, F. A, Tunc, C.,Saker, S. H, Lakshmanan, S. and Rakkiyappan, R. 2018. Application of delay differential equations in biological systems. Complexity, Vol. 2018.

Ruan, S. 2006. Delay differential equations in single species dynamics. Delay Differential Equation and Applications. Springer, Berlin.

Tsoularis A. and Wallace J. 2002. Analysis of logistic growth models. Mathematical Bioscience, 179(1), 21-55.

Yin, X. -B., Kumar, S. and Kumar, D. 2015. A modified homotopy analysis method for solution of fractional wave equations. Advances in Mechanical Engineering, 7(12). 